\title{
ASOCIACIÓN ENTRE RUMIACIONES Y MALESTAR EMOCIONAL EN PACIENTES RECIÉN DIAGNOSTICADOS DE CÁNCER
}

\section{ASSOCIATION BETWEEN RUMINATIONS AND EMOTIONAL DISTRESS IN PATIENTS NEWLY DIAGNOSED WITH CANCER}

\author{
Amador Priede1,2, César González-Blanch²,3, Andrés Herrán ${ }^{2,3}$ y Fernando Hoyuela ${ }^{2,3}$ \\ ' Asociación Española Contra el Cáncer. \\ 2 Servicio de Psiquiatría. Hospital Universitario Marqués de Valdecilla. Santander. \\ ${ }^{3}$ Unidad de investigación en Psiquiatría de Cantabria. Santander.
}

Resumen

Objetivo: El cáncer actúa como un potente estresor psicológico, generando malestar emocional en los pacientes. Los modos de procesamiento de la información basados en rumiaciones constituyen un importante factor de vulnerabilidad para la aparición de dicho malestar. Sin embargo, las rumiaciones no han sido convenientemente estudiadas en pacientes con cáncer. El presente estudio analiza la relación existente entre rumiaciones y los síntomas ansiosos y depresivos en pacientes recién diagnosticados de cáncer.

Método: Se evaluó una muestra de 71 pacientes oncológicos recién diagnosticados mediante la Escala de Ansiedad y Depresión Hospitalaria, la Escala de Respuestas Rumiativas, la Escala MOS de Apoyo Social y una entrevista clínica. Se analizaron las correlaciones entre rumiaciones, apoyo social, variables sociodemográficas y clínicas, y malestar emocional (tanto ansiedad como depresión). Se realizó un análisis de regresión múltiple con el fin de determinar las contribuciones específicas de cada variable en el modelo explicativo.

Resultados: La sintomatología ansiosa correlacionó con rumiaciones, apoyo social, sexo y edad. La sintomatología depresiva correlacionó con rumiaciones y apoyo social. En el análisis multivariado la sintomatología ansiosa se asoció con las dos subescalas de rumiacio-
Abstract

Objective: Cancer is a strong psychological stressor, generating emotional distress among patients. Rumination is an information processing mode that increases the vulnerability to such distress. However, rumination has not been appropriately studied in cancer patients. This research examines the relationship between rumination and anxiety/depression symptoms among recently diagnosed cancer patients.

Method: A sample of 71 recently diagnosed cancer patients was assessed through the Hospital Anxiety and Depression Scale, the Ruminative Responses Scale, the MOS Social Support Survey and a clinical interview. Correlations between ruminations (and its subscales of brooding and reflection), social support, clinical and socio-demographic variables and emotional distress (both anxiety and depression) were analyzed. Multiple regression models were performed, in order to determine the specific contribution of each variable in the explanatory model.

Results: Anxiety symptoms correlated with ruminations, social support, sex and age. Depression symptoms correlated with ruminations and social support. Multivariate models show that anxiety symptoms were associated with both rumination subscales and sex. Depression symptoms were associated with the brooding subscale.

\section{Correspondencia:}

Amador Priede.

Consultas de Psiquiatría. Hospital Universitario Marqués de Valdecilla.

39008 Santander. Cantabria.

Email: amadorpriede@gmail.com 
nes y con el sexo, mientras que los síntomas depresivos se asociaron con la subescala de reproches.

Conclusiones: Las rumiaciones se asocian con una mayor presencia de síntomas ansiosos y depresivos en pacientes con un diagnóstico reciente de cáncer, pudiendo constituir una diana terapéutica clave para la prevención del malestar emocional asociado al diagnóstico e inicio del tratamiento. Estudios longitudinales deben determinar el verdadero valor predictivo de las rumiaciones sobre el malestar emocional.

Palabras clave: rumiaciones, malestar emocional, cáncer, ansiedad, depresión, cognitivo.
Discussion: Ruminations are associated with higher levels of anxiety and depression symptoms among recently diagnosed cancer patients. Rumination become an important therapeutic target in prevention of psychological distress associated to both diagnosis and treatment initiation. Longitudinal research should be carry out in order to determine the predictive value of ruminations for psychological distress.

Key words: Rumination, psychological distress, cancer, anxiety, depression, cognitive.

\section{INTRODUCCIÓN}

El cáncer actúa como un potente estresor sobre los pacientes, contribuyendo a generar estados de malestar emocional. Los estudios que analizan la prevalencia de malestar emocional en pacientes con cáncer proporcionan datos diversos, variando las cifras en función de diferentes factores, como los instrumentos de medida y la metodología empleados. Por lo general, los estudios informan de prevalencias situadas entre el $20 \%$ y el $47 \%{ }^{(1-3)}$.

Sin embargo, el impacto del malestar emocional en estos pacientes ha sido subestimado, debido a la importancia de su patología somática y a la falta de recursos terapéuticos adecuados. El grado de incertidumbre provocado por la enfermedad, la preocupación por la muerte, las complicaciones físicas, el dolor, el cambio en la imagen corporal, etc, son estresores que pueden contribuir al desarrollo de dicho malestar ${ }^{(4)}$. Por ello, supone un gran reto el detectar este problema y tratarlo, especialmente cuando diferentes autores plantean que está infradiagnosticado e infratratado $^{(5,6)}$.
El modelo explicativo más ampliamente extendido para conceptualizar los problemas psicopatológicos, tanto en población general, como en pacientes con cáncer ha sido el modelo cognitivo de $\operatorname{Beck}^{(7)}$. Si bien esta teoría ha contado con una gran aceptación durante años, la evidencia científica más reciente ha llevado a reformular el modelo original añadiendo nuevos procesos y conceptos $^{(8)}$. De este modo, si originalmente se daba un mayor peso a los esquemas y contenidos negativos de la mente (pensamientos, emociones), los nuevos modelos explicativos hacen mayor énfasis en los modos de procesamiento cognitivo que emplea la persona ${ }^{(9)}$. En esta línea, uno de los desarrollos más importantes es el referido a los modos de procesamiento basados en rumiaciones.

Entendemos las rumiaciones como pensamientos repetitivos y pasivos acerca de los propios síntomas depresivos, así como sobre sus causas y consecuencias ${ }^{(10)}$. De este modo, al rumiar, las personas tienen repetidamente pensamientos del tipo "¿por qué no consigo avanzar?", "¿qué es lo que está mal en mí?", "no creo que nunca pueda salir de esto", sin que estos pensa- 
mientos conduzcan a estrategias dirigidas a resolver problemas. Además, los estudios encuentran que es una característica relativamente estable en los individuos ${ }^{(11)}$.

Según la teoría de estilos de respuesta, las rumiaciones, en el contexto de síntomas de depresión, exacerban y prolongan los mismos, aumentando la probabilidad de que se hagan crónicos y evolucionen hacia un cuadro de depresión mayor. Se han planteado cuatro mecanismos mediante los cuales las rumiaciones pueden influir y prolongar la depresión ${ }^{(12)}$. Primero, rumiar aumenta los efectos del ánimo deprimido sobre el pensamiento, facilitando el que se activen pensamientos y recuerdos negativos. Segundo, las rumiaciones interfieren con una adecuada resolución de problemas. Tercero, disminuye la motivación para iniciar conductas instrumentales. Y cuarto, mina las relaciones interpersonales, conllevando una pérdida del apoyo social.

Este tipo de pensamientos ocurren tanto en individuos sanos, como en pacientes, si bien en estos suelen encontrarse amplificados. De hecho, se considera que la rumiación es un proceso desadaptativo que puede mantener o iniciar una depresión, además de predecir los niveles futuros de ansiedad y depresión ${ }^{(13,14)}$.

Aunque la investigación sobre rumiaciones en pacientes con cáncer es aún reciente y existen pocos estudios en este campo, diferentes estudios encuentran que éstas se asocian de modo significativo con mayores niveles de malestar emocional ${ }^{(15)}$ o de sintomatología depresiva ${ }^{(16)}$. Comparados con sujetos sanos, los pacientes paliativos perciben las rumiaciones como menos controlables y generadoras de mayor malestar emocional, aunque difieren en su frecuencia o duración ${ }^{(17)}$.

Otros estudios sobre rumiaciones en pacientes con cáncer distinguen dos facetas diferenciadas de los pensamientos intrusivos $^{(18,19)}$. La primera es desadaptativa y consiste en un procesamiento pasivo sobre las causas de los síntomas y de los objetivos no logrados, llamado brooding (reproches). Este tipo de procesamiento es el que se asocia a mayores niveles de depresión y de síntomas de estrés postraumático. La segunda, adaptativa, Ilamada reflection (reflexión), consiste en un procesamiento activo que busca resolver problemas, y se asocia a menor nivel de depresión y a un mayor crecimiento postraumático.

Los instrumentos utilizados para la evaluación de las rumiaciones en estos pacientes han sido muy diversos, empleándose, la Escala de Respuestas Rumiativas ${ }^{(17)}$ (RRS), el Rumination Inventory ${ }^{(18)}(\mathrm{RI})$, la Cancer-Related Rumination Scale ${ }^{(19)}$ (CRRS) derivada del anterior, y la subescala de rumiación del Cognitive Emotion-Regulation Questionnaire $^{(15)}$ (CERQ).

La aún escasa investigación en este campo hace necesario ahondar en la comprensión de cómo las rumiaciones influyen en la adaptación psicológica de los pacientes con cáncer y en la aparición de malestar emocional. Por ello, en este estudio se contrasta la hipótesis de que mayores niveles de rumiaciones se asociarán a mayores niveles de distrés, tanto en forma de síntomas de depresión, como de ansiedad.

\section{MÉTODO}

\section{Ámbito de estudio y procedimiento}

El presente estudio forma parte de un proyecto de investigación más amplio, que estudia la influencia de variables cognitivas en la adaptación al cáncer. Este estudio se está Ilevando a cabo dentro de un proyecto de evaluación de necesidades y cribado de trastornos mentales en pacientes con cáncer recién diagnosticados que se lleva a cabo en el Hospital Universitario Marqués de Valdecilla (Santander, España). Dicho proyecto, denominado "Primer Im- 
pacto", ha sido diseñado por la Asociación Española Contra el Cáncer y se Ileva a cabo por medio de un convenio de colaboración con el hospital. Los facultativos de servicios implicados en la atención de pacientes con cáncer (Oncología Médica, Oncología Radioterápica, Hematología, Ginecología Oncológica y Hospital de Día Médico) realizan la derivación de los nuevos pacientes diagnosticados.

Tras realizar la consulta diagnóstica se oferta la participación en el estudio, informando verbalmente y resolviendo posibles dudas. En caso de decidir participar se pasa a firmar la hoja de consentimiento y se explica cómo deben cumplimentarse los cuestionarios. Todos ellos se responden de manera autoaplicada por el paciente, quien los contesta en su domicilio, para posteriormente entregarlos en el hospital.

Este estudio ha sido aprobado por el Comité de Ética de Investigación Clínica del Instituto de Formación e Investigación Marqués de Valdecilla (IFIMAV).

\section{Participantes}

En el estudio se han incluido pacientes con un primer diagnóstico de cáncer confirmado por estudio de Anatomía Patológica. Se excluyeron aquellos pacientes con un tiempo desde el diagnóstico mayor a 4 meses, menores de 18 años, mayores de 70, pacientes con recidivas o que hayan padecido otro proceso oncológico con anterioridad, pacientes con retraso mental, deterioro de las funciones cognitivas, o incapacidad para comprender las pruebas de evaluación.

Entre diciembre de 2011 y diciembre de 2012, se derivaron 150 pacientes al programa de los cuales 116 (77,33\%) cumplían los criterios de inclusión en el estudio. De estos, 12 rechazan participar. De los 104 que acceden a participar en el estudio, 71 $(68,27 \%)$ realizan la evaluación, entregando los cuestionarios cumplimentados.

\section{Materiales}

El malestar emocional se evaluó mediante la Hospital Anxiety and Depression Scale ${ }^{(20,21)}$ (HADS). Es una escala que consta de 14 ítems, divididos en dos subescalas de Depresión y Ansiedad. Es el instrumento de referencia a la hora de evaluar niveles de depresión y ansiedad en pacientes con patologías somáticas. La consistencia interna de la escala global es alta $(\alpha=0,90)$, así como la de sus subescalas (Ansiedad $\alpha=0,85$ y Depresión $\alpha=0,84$ ).

Las rumiaciones se evaluaron mediante la Escala de Respuestas Rumiativas ${ }^{(22,23)}$ (Ruminative Responses Scale; RRS). Esta escala consta de 22 ítems de tipo Likert con cuatro niveles de respuesta. La consistencia interna de la escala es alta $(\alpha=0,93)$. Algunos autores ${ }^{(24)}$ sugieren que existe cierto solapamiento entre algunos ítems de este cuestionario y la sintomatología depresiva. El análisis factorial de los ítems no asociados directamente con depresión deja una estructura de dos factores ${ }^{(25)}$, reflection (reflexión) y brooding (reproches), anteriormente citados, con adecuada fiabilidad ( $\alpha=0, .80$ y $\alpha=0,74$ respectivamente).

El apoyo social es una variable determinante en la adaptación al cáncer ${ }^{(26,27)}$ y por ello se hace necesario controlar su efecto. Dicha variable se ha evaluado mediante el cuestionario MOS de apoyo social(28) (MOS Social Support Survey; MOS-SSS). Esta escala está formada por 19 ítems de tipo Likert con cinco niveles de respuesta más un ítem que evalúa la red social. La escala ha sido validada en castellano para pacientes con enfermedades crónicas $^{(29)}$ y cáncer ${ }^{(30)}$. La consistencia interna de sus tres subescalas (apoyo emocional/informacional, apoyo afectivo y apoyo instrumental) es adecuada, encontrándose entre $\alpha=0,94-0,75$.

Se analiza el efecto de distintas variables sociodemográficas (sexo, edad, nivel socioeducativo, estado civil) y clínicas 
(diagnóstico, tiempo desde diagnóstico, tiempo desde inicio de tratamiento, antecedentes psicopatológicos), recogidas mediante un cuestionario elaborado ad hoc.

\section{Análisis estadístico}

Los análisis estadísticos se han Ilevado a cabo mediante el software Statistical Package for the Social Sciences (SPSS 19). Los cálculos realizados a priori para determinar el tamaño muestral ${ }^{(31)}$ requerido indican que para detectar un tamaño del efecto moderado $(r=0,30, \alpha=0, .05)$ con una potencia estadística de 0,8 serán necesarios 67 sujetos.

Se realizó un análisis descriptivo de la muestra, mostrando las medias y desviaciones típicas de las variables sociodemográficas y clínicas más relevantes. En el caso de variables nominales se presenta el número de casos y su porcentaje sobre el total. Se evaluaron las posibles diferencias entre los sujetos que realizan la evaluación completa y aquellos que rehúsan (tanto los que rechazan participar, como quienes no entregan los cuestionarios), por medio de pruebas $t$ para diferencias de medias, y chi $^{2}$ o phi en caso de que las variables fueran nominales.

Se analiza la asociación entre las variables de interés y las variables ansiedad y depresión por medio del coeficiente de correlación de Pearson. Se empleó el coeficiente de correlación biserial-puntual para las variables dicotómicas.

Con el fin de determinar si las rumiaciones explican la presencia de síntomas ansiosos y/o depresivos, se realizó un análisis de regresión múltiple (stepwise), incluyendo, como variables predictoras aquellas cuyo coeficiente de correlación alcance una significación inferior a .1. En un primer bloque se introdujeron las posibles variables mediadoras y en el segundo las dos subescalas de rumiaciones.

\section{RESULTADOS}

\section{Características sociodemográficas}

De los pacientes derivados al programa, 116 cumplían los criterios de inclusión en el estudio. La Tabla 1 muestra las características sociodemográficas y clínicas de los sujetos incluidos, frente a aquellos que rechazaron participar y quienes no realizaron la evaluación. Estos grupos no presentan diferencias significativas en ninguna de estas variables, excepto en antecedentes psicopatológicos, encontrándose porcentualmente, el doble de personas con antecedentes en el grupo de participantes.

En la Tabla 2 se muestran las medias y desviaciones típicas de las principales variables analizadas.

\section{Análisis bivariado}

En el análisis bivariado se evidenció que la sintomatología ansiosa correlaciona con la escala total de rumiaciones $(r=0,81, p<0,001)$, la subescala de reproches $(r=0,67, p<0,001)$, la subescala de reflexión $(r=0,63, p<0,001)$, apoyo social $(r=-$ $0,43, p=0,001)$, sexo femenino $\left(r_{\mathrm{bp}}=0,35\right.$, $p=0,003)$ y edad $(r=-0,27, p=0,024)$.

La sintomatología depresiva correlacionó con la escala total de rumiaciones $(r=0,73, p<0,001)$, la subescala de reproches $(r=0,59, p<0,001)$, la subescala de reflexión $(r=0,52, p<0,001)$ y apoyo social $(r=-0,40, p=0,001)$.

Por sexos, las mujeres presentaron un nivel de rumiaciones más elevado en la escala total $\left(t_{62.879}=-20,46 ; p=0,016\right) 0$, Sin embargo, no mostraron diferencias en la subescala de reproches $\left(t_{68}=-10,20 ; p=0,235\right)$, ni en la de reflexión $\left(t_{68}=-10,44 ; p=0,156\right)$.

\section{Análisis multivariado}

En el análisis multivariado la sintomatología ansiosa se asoció con la variable 


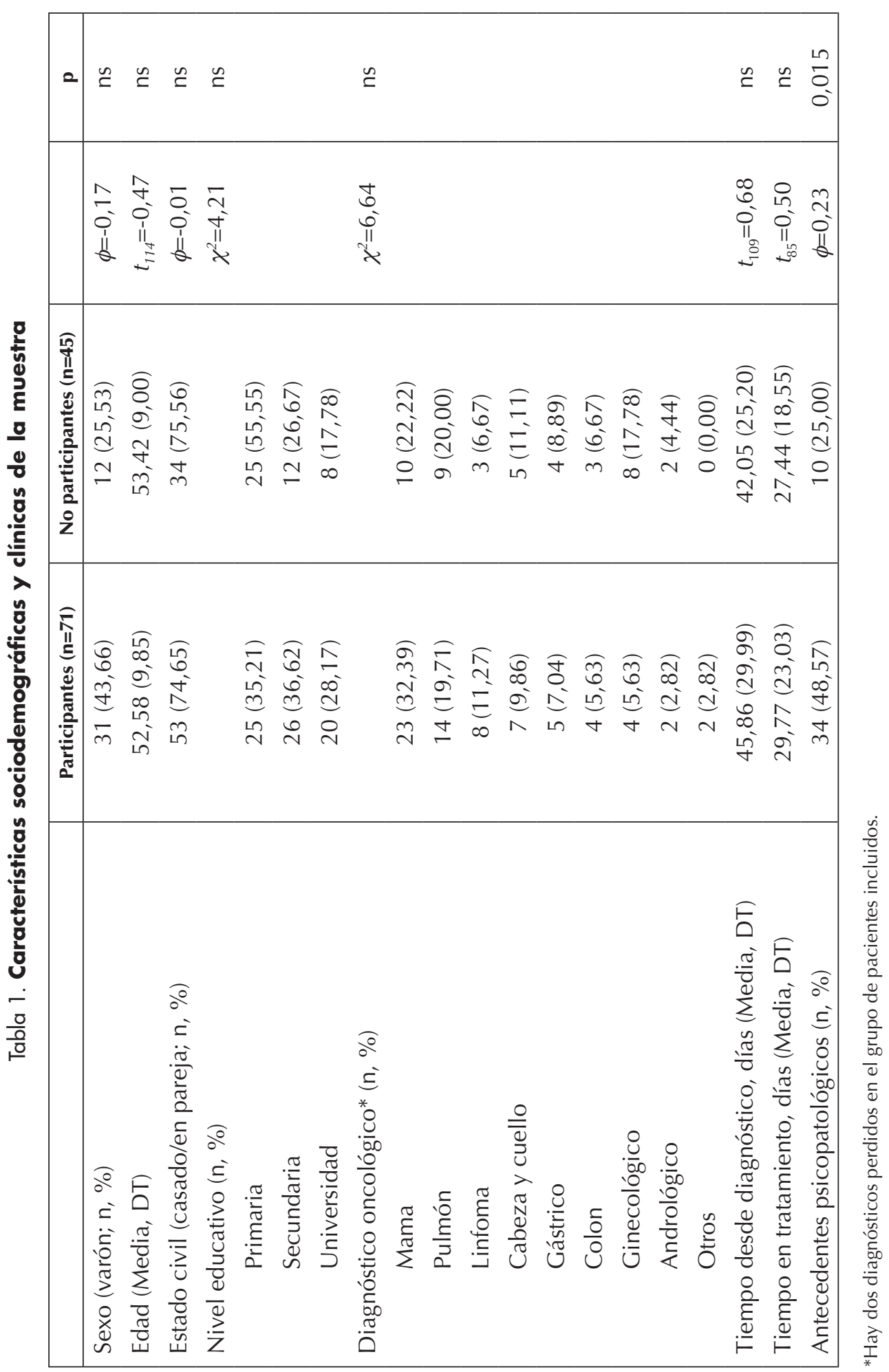


Tabla 2. Estadísticos descriptivos de las escalas empleadas

\begin{tabular}{|l|c|c|}
\cline { 2 - 3 } \multicolumn{1}{c|}{} & Media & DT \\
\hline HADS-A & 7,31 & 4,34 \\
\hline HADS-D & 4,56 & 4,03 \\
\hline RRS-Total & 36,64 & 12,63 \\
\hline RRS-Reproches & 8,80 & 3,59 \\
\hline RRS-Reflexión & 7,60 & 2,63 \\
\hline MOS & 84,62 & 12,13 \\
\hline
\end{tabular}

HADS-A, subescala de ansiedad; HADS-D, subescala de depresión; RRS, escala de respuestas rumiativas; RRS-Reproches, subescala de reproches; RRS-Reflexión, subescala de reflexión; MOS, escala de apoyo social.

Tabla 3. Modelo de regresión múltiple para explicar los síntomas de ansiedad

\begin{tabular}{|l|c|c|c|c|}
\hline & B & SE(B) & Beta & p \\
\hline (Constante) & 0,90 & 3,88 & & 0,817 \\
\hline Apoyo social & $-0,05$ & 0,04 & $-0,13$ & 0,194 \\
\hline Sexo & 1,76 & 0,77 & 0,21 & 0,026 \\
\hline RRS-Reflexión & 0,56 & 0,23 & 0,35 & 0,018 \\
\hline RRS-Reproches & 0,36 & 0,18 & 0,30 & 0,043 \\
\hline
\end{tabular}

Adj. $R^{2}=0,52 ; F(4,60)=18,37 ; p<0,001$

Tabla 4. Modelo de regresión múltiple para explicar los síntomas de depresión

\begin{tabular}{|l|c|c|c|c|}
\hline & B & SE(B) & Beta & p \\
\hline (Constante) & 4,26 & 3,80 & & 0,267 \\
\hline Apoyo social & $-0,58$ & 0,04 & $-0,17$ & 0,125 \\
\hline RRS-Reproches & 0,60 & 0,13 & 0,52 & $<0,001$ \\
\hline
\end{tabular}

Adj. $R^{2}=0,36 ; F(2,62)=18,60, p<0,001$

sexo, y las subescalas de reflexión y reproches (Tabla 3), mientras que los síntomas depresivos se asociaron únicamente con la subescala de reproches (Tabla 4).

\section{DISCUSIÓN}

El presente estudio confirma la asociación entre rumiaciones y sintomatología ansiosa y depresiva en pacientes oncológi- cos de diagnóstico reciente. Según evidencian los modelos multivariados, aquellos pacientes de sexo femenino, con mayor nivel de rumiaciones, en sus dos vertientes de reflexión y reproches es más probable que presenten sintomatología ansiosa. Por otro lado, aquellos pacientes que presentan un mayor nivel de rumiaciones, en su vertiente de reproches, es más probable que presenten síntomas de depresión. El 
modelo multivariado para ansiedad explica el $52 \%$ de la varianza, mientras que el de depresión explica el 36\%.

Las rumiaciones se presentan como la variable más fuertemente asociada tanto con sintomatología ansiosa como depresiva, además de ser la única variable que tiene poder explicativo sobre ambas, según los modelos multivariados. Esto concuerda con la literatura previa, en la que aparece como un predictor o mediador significativo del malestar emocional, tanto en población general, como en pacientes oncológicos $^{(15-17)}$. En esta muestra, tanto los reproches como la reflexión se asocian con mayores niveles de malestar emocional, contrariamente a lo planteado en algunos estudios $^{(18,19)}$ en los que el componente reflexión de las rumiaciones no influye sobre el malestar.

Por sexos, las mujeres presentan un mayor nivel de rumiaciones que los varones. Sin embargo, cuando se analizan las subescalas de reproches y reflexión, en las cuales se eliminan los ítems de la escala total que pueden confundirse con síntomas depresivos, esta diferencia desaparece.

El apoyo social, de acuerdo con la literatura previa ${ }^{(26,27)}$, se asocia con los niveles de ansiedad y depresión, aunque no alcanza la significación en los modelos multivariados.

La principal limitación del presente estudio consiste en su diseño transversal, el cual impide establecer relaciones causales entre las variables. Por ello se hace necesario realizar un seguimiento longitudinal de los pacientes, con el fin de determinar si las variables estudiadas tienen poder predictivo sobre la sintomatología ansiosa y depresiva al cabo del tiempo. Es especialmente interesante valorar esta evolución tras haberse producido la finalización de los tratamientos oncológicos.

Otro factor limitante es el aún reducido tamaño muestral. Si bien se alcanza la cifra necesaria para detectar efectos moderados a nivel bivariado, se hace necesario aumentar el tamaño muestral de cara a cumplir los requerimientos de los análisis multivariados.

Aunque los pruebas psicométricas empleadas presentan una adecuada fiabilidad y validez, al ser varias de ellas de tipo autoinforme, pueden ser vulnerables a sesgos de respuesta (p.ej. tendencia central, restricción del rango, deseabilidad social, etc.). Además, el estudio presenta ciertos sesgos de selección que se evidencian por el porcentaje aumentado de participantes con antecedentes psicopatológicos entre aquellos que aceptaron participar en el estudio, aunque no existen diferencias en las demás características comparadas en la muestra.

Los datos de este estudio indican que la intervención psicológica especializada para el manejo de rumiaciones puede ser clave para la prevención del malestar emocional en los pacientes con un diagnóstico reciente de cáncer. En este sentido, algunas intervenciones como la terapia de aceptación y compromiso ${ }^{(32)}$, mindfulness ${ }^{(33,34)} \mathrm{O}$ la terapia metacognitiva ${ }^{(35)}$, aportan resultados prometedores de cara a disminuir las rumiaciones.

\section{Agradecimientos}

Este estudio ha sido financiado por medio de la Asociación Española Contra el Cáncer y del proyecto Talento Solidario de la Fundación Botín.

\section{REFERENCIAS BIBLIOGRÁFICAS}

1. Gil Moncayo FL, Costa Requena G, Perez FJ, Salamero M, Sanchez N, Sirgo A. Adaptación psicológica y prevalencia de trastornos mentales en pacientes con cáncer. Med Clin (Barc) 2008;130(3):90-2.

2. Massie MJ. Prevalence of depression in patients with cancer. J Natl Cancer Inst 
Monogr 2004;32:57-71. Doi: 10.1093/jncimonographs/lgh014

3. Derogatis LR, Morrow GR, Fetting J, Penman D, Piasetsky S, Schmale AM, et al. The prevalence of psychiatric disorders among cancer patients. JAMA 1983;249(6):751-7. Doi: 10.1001/jama.249.6.751

4. Shi Q, Smith TG, Michonski JD, Stein KD, Kaw C, Cleeland CS. Symptom burden in cancer survivors 1 year after diagnosis: A report from the American Cancer Society's Studies of Cancer Survivors. Cancer 2011;117(12):2779-90. Doi: 10.1002/ cncr.26146

5. Passik SD, Dugan W, McDonald MV, Rosenfeld B, Theobald DE, Edgerton S. Oncologists' recognition of depression in their patients with cancer. J Clin Oncol 1998;16(4):1594-600.

6. Massie MJ, Holland JC. Diagnosis and treatment of depression in the cancer patient. J Clin Psychiatry 1984;45(3 Pt 2):259.

7. BeckAT. Thinking and depression. I. Idiosyncratic content and cognitive distortions. Arch Gen Psychiatry 1963;9:324-33. Doi: 10.1001/archpsyc.1963.01720160014002

8. Vázquez C, Hernangómez L, Hervás G. Modelos cognitivos de la depresión: Su aplicación al estudio de las fases tempranas. In: Vázquez-Barquero JL, editor. Las fases tempranas de las enfermedades mentales: trastornos depresivos. Barcelona: Masson \& Elsevier; 2008.p.21-34.

9. Wilson KG, Luciano MC. El trastorno de evitación experiencial. Evidencias de los efectos del control evitativo de los eventos privados. In: Wilson KG, Luciano MC, editors. Terapia de Aceptación y Compromiso (ACT) Un tratamiento conductual orientado a los valores. Madrid: Pirámide; 2007.p.79-83.

10. Nolen-Hoeksema S. Responses to depression and their effects on the duration of depressive episodes. J Abnorm Psychol 1991;100(4):569-82. Doi:10.1037//0021843X.100.4.569
11. Nolen-Hoeksema S, Davis CG. "Thanks for sharing that": ruminators and their social support networks. J Pers Soc Psychol 1999;77(4):801-14. Doi:10.1037//00223514.77.4.801

12. Matthews G, Wells A. Rumination, depression, and metacognition: the S-REF model. In: Papageorgiou C, Wells A, editors. Depressive rumination: Nature, theory and treatment. Chichester: John Wiley \& Sons Ltd; 2004.p.125-51.

13. Nolen-Hoeksema S. The role of rumination in depressive disorders and mixed anxiety/ depressive symptoms. J Abnorm Psychol 2000;109(3):504-11. Foi:10.1037//0021843X.109.3.504

14. Nolen-Hoeksema S, Parker LE, Larson J. Ruminative coping with depressed mood following loss. J Pers Soc Psychol 1994;67(1):92-104. Doi:10.1037//00223514.67.1.92

15. Schroevers M, Kraaij V, Garnefski N. How do cancer patients manage unattainable personal goals and regulate their emotions? Br J Health Psychol 2008;13(Pt 3):551-62. Doi:10.1348/135910707X241497

16. Labelle L, Campbell T, Carlson L. Mindfulness-based stress reduction in oncology: evaluating mindfulness and rumination as mediators of change in depressive symptoms. Mindfulness 2010;1(1):28-40. Doi:10.1007/s12671-010-0005-6

17. Galfin JM, Watkins ER. Construal level, rumination, and psychological distress in palliative care. Psychooncology 2012;21(6):680-3. Doi:10.1002/pon.1948

18. Morris BA, Shakespeare-Finch J. Rumination, post-traumatic growth, and distress: structural equation modelling with cancer survivors. Psychooncology 2011;20(11):1176-83. Doi:10.1002/ pon. 1827

19. Chan MW, Ho SM, Tedeschi RG, Leung $\mathrm{CW}$. The valence of attentional bias and cancer-related rumination in posttraumatic stress and posttraumatic growth among women with breast cancer. Psychooncology 
2011;20(5):544-52. doi: 10.1002/pon.1761

20. Zigmond AS, Snaith RP. The hospital anxiety and depression scale. Acta Psychiatr Scand 1983;67(6):361-70. Doi: 10.1111/j.1600-0447.1983.tb09716.x

21. Herrero MJ, Blanch J, Peri JM, De Pablo J, Pintor L, Bulbena A. A validation study of the hospital anxiety and depression scale (HADS) in a Spanish population. Gen Hosp Psychiatry 2003 ;25(4):277-83. Doi;10.1016/S0163-8343(03)00043-4

22. Nolen-Hoeksema S, Morrow J. A prospective study of depression and posttraumatic stress symptoms after a natural disaster: the 1989 Loma Prieta Earthquake. J Pers Soc Psychol 1991;61(1):115-21. Doi:10.1037//0022-3514.61.1.115

23. Hervás G. Adaptación al castellano de un instrumento para evaluar el estilo rumiativo: la escala de respuestas rumiativas. Rev Psicopatol Psicol Clín 2008;13(2):111-21.

24. Roberts J, Gilboa E, Gotlib I. Ruminative response style and vulnerability to episodes of dysphoria: gender, neuroticism, and episode duration. Cognit Ther Res 1998;22(4):401-23.

25. Treynor W, Gonzalez R, Nolen-Hoeksema S. Rumination reconsidered: A Psychometric analysis. Cognit Ther Res 2003;27(3):247-59.

26. Eom CS, Shin DW, Kim SY, Yang HK, Jo HS, Kweon SS, et al. Impact of perceived social support on the mental health and health-related quality of life in cancer patients: results from a nationwide, multicenter survey in South Korea. Psychooncology. 2012 Jul 26. Doi: 10.1002/ pon.3133

27. Luszczynska A, Pawlowska I, Cieslak R, Knoll N, Scholz U. Social support and quality of life among lung cancer patients: a systematic review. Psychooncology. 2012 Oct 25. Doi: 10.1002/pon.3218
28. Sherbourne CD, Stewart AL. The MOS social support survey. Soc Sci Med. 1991; 32(6):705-14. Doi: 10.1016/02779536(91)90150-B

29. Revilla Ahumada L, Luna del Castillo J, Bailon Munoz E, Medina MI. Validacion del cuestionario MOS de apoyo social en atencion primaria. Medicina de Familia (And). 2005;6(1):10-8.

30. Costa Requena G, Salamero M, Gil F. [Validity of the questionnaire MOS-SSS of social support in neoplastic patients]. Med Clin (Barc) 12;128(18):687-91.

31. Hervada Vidal X, Santiago Pérez MI, Vázquez Fernández E, Castillo Salgado C, Loyola Elizondo E, Silva Ayçaguer LC. Epidat 3.0 programa para análisis epidemiológico de datos tabulados. Revi Esp Salud Pública 2004;78(2):277-80.

32. Feros DL, Lane L, Ciarrochi J, Blackledge JT. Acceptance and Commitment Therapy (ACT) for improving the lives of cancer patients: a preliminary study. Psychooncology 2013;22(2):459-64.

33. Hoffman CJ, Ersser SJ, Hopkinson JB, Nicholls PG, Harrington JE, Thomas PW. Effectiveness of mindfulness-based stress reduction in mood, breast- and endocrine-related quality of life, and well-being in stage 0 to III breast cancer: A randomized, controlled trial. J Clin Oncol 2012;30(12):1335-42.

34. Lengacher CA, Johnson-Mallard V, PostWhite J, Moscoso MS, Jacobsen PB, Klein TW, et al. Randomized controlled trial of mindfulness-based stress reduction (MBSR) for survivors of breast cancer. Psychooncology. 2009;18(12):1261-72.

35. Wells A, Papageorgiou C. Metacognitive therapy for depressive rumination. In: $\mathrm{Pa}$ pageorgiou C, Wells A, editors. Depressive rumination: nature, theory and treatment. Chichester: John Wiley \& Sons Ltd; 2004. 